\title{
Over Absolute Valued Algebras with Central Element not Necassary Idempotent
}

\author{
Alassane Diouf ${ }^{1}$, André S. Diabang ${ }^{1}$, Alhousseynou Ba ${ }^{1}$ \& Mankagna A. Diompy ${ }^{1}$ \\ ${ }^{1}$ Département de Mathématiques et Informatiques, Faculté des Sciences et Techniques, Université Cheikh Anta Diop, \\ Dakar, Sénégal \\ Correspondence: Alassane Diouf, Département de Mathématiques et Informatiques, Faculté des Sciences et Techniques, \\ Université Cheikh Anta Diop, Dakar, Sénégal. E-mail: dioufalassane@hotmail.fr
}

Received: December 28, 2014 Accepted: February 4, 2017 Online Published: March 23, 2017

doi:10.5539/jmr.v9n2p123 URL: https://doi.org/10.5539/jmr.v9n2p123

\begin{abstract}
We study the absolute valued algebras containing a central element non necessary idempotent. We determine the absolute valued algebras containing a central element if we add some requirements. Also we gives a classification of finitedimensional absolute valued algebras containing a generalized left unit and central element.
\end{abstract}

Keywords: Absolute valued algebra, central element, left unit and generalized left unit.

Mathematics Subject Classification: 17A35, 17A36

\section{Introduction}

The absolute valued algebras are introduced by Ostrowski in 1918. It's the normed algebra $A$ such that $\|x y\|=\|x\|\|y\|$ for all $x, y$ in $A$. An algebra is called division if and only if $R_{x}$ and $L_{x}$ are bijective for all $x$ in $A$. The category of finite-dimensional absolute valued algebra is a full subcategory of the category of division algebra. If $A$ is a finite dimensional absolute valued algebra, then $A$ has dimension 1, 2, 4 or 8 (Bott, et al., 1958; Kervaire, 1958), $A$ is isotopic to $\mathbb{R}, \mathbb{C}, \mathbb{H}$ or $\mathbb{O}$ and the norm of $A$ comes from an inner product(Albert, 1947). We have in (Beslimane \& Moutassim, 2011; Diankha, et al., 2013) a classification of absolute valued algebras with left unit and containing a central element. The norm of absolute valued algebra containing a central idempotent $c$, comes from to an inner product and the isometric map $x \mapsto x^{\star}:=2(x \mid c) c-x$ is an involution (El-Mallah, 1990). For $\|u\|=1$, we recall the following notations $\mathbb{H}_{u}:=\mathbb{H}_{T_{u, \bar{u}}}$, and $\mathbb{O}_{u}:=\mathbb{O}_{T_{u, \bar{u}}}$. Let $a, b \in \mathbb{H}$ such that $\|a\|=\|b\|=1$, we recall that $\mathbb{H}(a, b):=\left(\mathbb{H}, \star_{1}\right)$, with $x \star_{1} y=a x y b$ and ${ }^{\star} \mathbb{H}(a, b):=\left(\mathbb{H}, \star_{2}\right)$, with $x \star_{2} y=\bar{x} a y b$ (Ramirez, 1999). Let $A$ be an algebra, we note that $Z(A)=\{a \in A: a x=x a$ for all $x \in A\}$. In this work we give a characterization of finite dimensional absolute valued algebra containing a central element. We determine the finite-dimensional absolute algebra containing a genaralized left unit and central element. We classify the absolute valued algebra containing a central element if we add some conditions.

\section{Preliminary}

Let $f, g, f^{\prime}, g^{\prime}$ be linear isometries of euclidean space $\mathbb{A} \in\{\mathbb{R}, \mathbb{C}, \mathbb{H}, \mathbb{O}\}$ fixing 1 , and let $\Phi: \mathbb{A} \rightarrow \mathbb{A}$ be a linear mapping. Then it is easy to see that $\Phi: \mathbb{A}_{f, g} \rightarrow \mathbb{A}_{f^{\prime}, g^{\prime}}$ is an algebra isomorphism fixing 1 if and only if $\Phi: \mathbb{A} \rightarrow \mathbb{A}$ is an algebra automorphism and $\left(f^{\prime}, g^{\prime}\right)=\left(\Phi \circ f \circ \Phi^{-1}, \Phi \circ g \circ \Phi^{-1}\right)$ (Calderon, et al., 2011)

Let $\mathbb{A}$ be one of the unital absolute valued algebras $\mathbb{R}, \mathbb{C}, \mathbb{H}$ of dimension $m$. Consider the caley dickson product $\odot$ in $\mathbb{A} \times \mathbb{A}$, we define on the space $\mathbb{A} \times \mathbb{A}$ the product

$$
(x, y) \star\left(x^{\prime}, y^{\prime}\right)=\left(f_{1}(x), f(x)\right) \odot\left(g_{1}\left(x^{\prime}\right), g\left(y^{\prime}\right)\right) .
$$

With $f_{1}, g_{1}, f, g$ be linear isometries of $\mathbb{A}$ and $f_{1}(1)=g_{1}(1)=1$. We obtain a $2 m$-dimensional absolute valued real algebra $\mathbb{A} \times \mathbb{A}_{\left(f_{1}, f\right),\left(g_{1}, g\right)}$. The process is called the duplication process (Calderon, \& et al., 2011). Note that the algebra is left unit if $g_{1}=g=I_{\mathbb{A}}$ and this case we note the algebra by $\mathbb{A} \times \mathbb{A}_{\left(f_{1}, f\right)}$ (Rochdi, 2003).

Theorem 1 The finite-dimensional absolute valued real algebras with left unit are precisely those of the form $\mathbb{A}_{\varphi}$, where $\mathbb{A} \in\{\mathbb{R}, \mathbb{C}, \mathbb{H}, \mathbb{O}\}$ and $\varphi$ is an isometric of the euclidien espace $\mathbb{A}$ fixed 1 , and $\mathbb{A}_{\varphi}$ denotes the absolute valued real algebra obtained by endowing the normed space of $\mathbb{A}$ with the product $x \odot y:=\varphi(x) y$. Moreover, given linear isometries $\varphi, \phi: \mathbb{A} \rightarrow \mathbb{A}$ fixing 1 , the algebras $\mathbb{A}_{\varphi}$ and $\mathbb{A}_{\phi}$ are isomorphic if and only if there exists an algebra automorphism $\psi$ of $\mathbb{A}$ satisfying $\phi=\psi \circ \varphi \circ \psi^{-1}$ ((Rochdi, 2003)). 


\section{Finite Dimensional Absolute Valued Algebra Containing a Central Element}

An element $c$ in $A$ is called central if $L_{c}=R_{c}$. In this paragraph, the central element is non necessary idempotent. As $A$ isalternative, Artin's theorem (Schafer, 1996) shows that for any $x, y \in A$, the set $\{x, y, \bar{x}, \bar{y}\}$ is contained in an associative subalgebra of $A$.

Theorem 2 Let $A$ be an finite dimensional absolute valued algebra with nonzero central element $c$. Then $A$ is precisely $\mathbb{R}$, $\mathbb{C}, \stackrel{\star}{\mathbb{C}}$ or of the form $\mathbb{A}_{\varphi, \psi}$, with $\mathbb{A}=\{\mathbb{H}, \mathbb{O}\}, \varphi$ a linear isometry of the euclidien space $\mathbb{A}$ fixing 1 and $\psi=L_{\overline{\varphi(c)}} \circ R_{\varphi(c)} \circ \varphi$. Moreover for $\operatorname{dim}(A) \geq 4$, if $\psi=I_{A}$, then $A$ is isomorphic to $\mathbb{H}(c, 1)$ or $\mathbb{O}_{c}$.

Proof. If $\operatorname{dim}(A) \leq 2$, the result is clear. Assume now $\operatorname{dim}(A) \geq 4$. Then the algebra $A$ is of the form $\mathbb{A}_{\varphi, \psi}$, where $\psi, \varphi$ are the linear isometries of the euclidien space $\mathbb{A} \in\{\mathbb{H}, \mathbb{O}\}$ such that $\psi(1)=\varphi(1)=1$ (Calderon, at al., 2011). Using now $x \odot c=c \odot x$, for all $x$ in $\mathbb{A} \Leftrightarrow \varphi(x) \psi(c)=\varphi(c) \psi(x)$, for all $x$ in $\mathbb{A}$.

For $x=1$, we have $\psi(c)=\varphi(c)$.

$$
\begin{aligned}
\varphi(x) \psi(c)=\varphi(c) \psi(x), \text { for all } x \text { in } \mathbb{A} & \Rightarrow \varphi(x) \varphi(c)=\varphi(c) \psi(x), \text { for all } x \text { in } \mathbb{A} \\
& \Rightarrow \psi(x)=\overline{\varphi(c)} \varphi(x) \varphi(c), \text { for all } x \text { in } \mathbb{A} \\
& \Rightarrow \psi(x)=\left(L_{\overline{\varphi(c)}} \circ R_{\varphi(c)} \circ \varphi\right)(x), \text { for all } x \text { in } \mathbb{A} \\
& \Rightarrow \psi=L_{\overline{\varphi(c)}} \circ R_{\varphi(c)} \circ \varphi
\end{aligned}
$$

Moreover if $\psi=I_{A}$, then $A$ is left unit and $\varphi=L_{c} \circ R_{\bar{c}}$ (Diankha, et al., 2013). For the algebra $\mathbb{H}_{c}$, we have the following isomorphism of algebra $\Phi: \mathbb{H}(c, 1) \rightarrow \mathbb{H}_{c} \quad x \mapsto x c$.

Theorem 3 Let $A$ be an finite-dimensional absolute valued algebra containing a central idempotent $c$. Then $c \in\{1\} \cup$ $\left\{-\frac{1}{2}+u: u \in \operatorname{Im}(\mathbb{A})\right.$ and $\left.\|u\|=\frac{\sqrt{3}}{2}\right\}$.

Proof. Using Theorem 3.3., $A$ is of the form $\mathbb{A}_{\varphi, \psi}$, where $\varphi$ is a linear isometric of $\mathbb{A}$ fixing 1 and $\psi=L_{\overline{\varphi(c)}} \circ R_{\varphi(c)} \circ \varphi$. We remark also $\varphi(c)=\psi(c)$, hence $c \odot c=\varphi(c) \psi(c)=\varphi(c)^{2}$. Assume now $c=\alpha+u \in S(\mathbb{A})$ (with $\mathbb{A}=\mathbb{R} \oplus \operatorname{Im}(\mathbb{A})$ : Frobenius decomposition). We note that if $u \in 1^{\perp}=\operatorname{Im}(\mathbb{A}),<1, \varphi(u)>=<\varphi(1), \varphi(u)>=<1, u>=0$. Hence we have $\varphi^{n}\left(1^{\perp}\right) \subseteq 1^{\perp}$, with $n \in \mathbb{N}$ and $\varphi\left(1^{\perp}\right)^{n} \in \mathbb{R}$ if and only if $n \in 2 \mathbb{N}$.

Hence $c \odot c=c$ and $\|c\|=1$ are equivalent to $\left\{\begin{array}{cc}\alpha^{2}+\varphi(u)^{2}= & \alpha \\ 2 \alpha \varphi(u)= & u \\ \|\varphi(c)\|= & 1\end{array}\right.$

The assertions (2) and (3) imply that $\frac{1}{4 \alpha^{2}} u^{2}=\alpha^{2}-1$ (4). Otherwise the assertions (1) and (2) implies that $\alpha^{2}+\left(\frac{1}{2 \alpha} u\right)^{2}=\alpha$ (5). The equality between (4) and (5) gives $\alpha=1$ or $-\frac{1}{2}$.

If $\alpha=1$, this is equivalent to $c=1$.

Assume now $\alpha=-\frac{1}{2}$, hence $c=-\frac{1}{2}+u$. Then

$$
\begin{aligned}
\|c\|^{2} & =\left\langle-\frac{1}{2}+u,-\frac{1}{2}+u\right\rangle \\
& =<-\frac{1}{2},-\frac{1}{2}>+\langle u, u\rangle \\
& =\frac{1}{4}+\|u\|^{2} \\
& =1
\end{aligned}
$$

This implies that $\|u\|=\frac{\sqrt{3}}{2}$.

Lemma 1 Let $A$ be an absolute valued algebras containing a nonzero central element $c$. The following assertions are equivalent:

1. $x^{2} c=x^{2}$, for all $x \in A$

2. A is finite dimensional and is isomorphic to $\mathbb{R}, \mathbb{C}, \mathbb{H}$ or $\mathbb{O}$.

Proof. 2) $\Rightarrow$ 1) is clear.

Now assume 1), Using the equality $(x+c)^{2} c=(x+c)^{2}$ for all $x$ in $A$, we have $(x c-x) c=0$ for all $x$ in $A$. Then $L_{c}=R_{c}=I_{A}$ and $A$ is isomorphic to $\mathbb{R}, \mathbb{C}, \mathbb{H}$ or $\mathbb{O}$ (Urbanik \& Wright, 1960). 
The group $G_{2}$ acts transitively on the sphere $S(\operatorname{Im}(\mathbb{O})):=S^{6}$, that is the mapping $G_{2} \rightarrow S^{6} \Phi \mapsto \Phi(i)$ is surjective (Postnikov, 1985).

Definition 1 An element $e \in A$ is called strongly left unit, if it's left unit and square root of right unit: $L_{e}=R_{e}^{2}=I_{A}$ (Diouf, 2017).

Theorem 4 Let $A$ be an absolute valued algebra with strongly left unit and containing a central element $c$. Then $c \in$ $S(\mathbb{R}) \cup 1^{\perp}$ and $A$ is finite dimensional and isomorphic to $\mathbb{R}, \mathbb{C}, \mathbb{H}, \mathbb{H}(i, 1)$, $\mathbb{O}$ or $\mathbb{O}_{i}$.

Proof. It's clear that $A$ is of finite dimensional. If $\operatorname{dim}(A) \leq 2$, the result is clear that $A$ is isomorphic to $\mathbb{R}$ or $\mathbb{C}$. Assume now $\operatorname{dim}(A) \geq 4$ and $A$ contains a central element $c$ (Diankha, et al., 2013) proves that $A$ is of the form $\mathbb{A}_{\varphi}$, where $\mathbb{A} \in\{\mathbb{H}, \mathbb{O}\}, c \in S(\mathbb{A})$ and $\varphi=L_{c} \circ R_{\bar{c}}$.

Otherwise we have $R_{e}^{2}=I_{A} \Leftrightarrow(x \odot 1) \odot 1=x$. Hence

$$
\begin{aligned}
x & =(x \odot 1) \odot 1 \\
& =c(c x \bar{c}) \bar{c} \\
& =c^{2} x \bar{c}^{2} \text { Artin's theorem }
\end{aligned}
$$

The equality $c^{2} x=x c^{2}$, implies that $c^{2} \in S(\mathbb{R})=\{-1,1\}$.

If $c^{2}=1$, then $c= \pm 1$ and $A$ is isomorphic to $\mathbb{H}$ or $\mathbb{O}$.

If $c^{2}=-1$, then $c \in S(\operatorname{Im}(\mathbb{A}))$.

There exists $u \in S(\operatorname{Im}(\mathbb{A}))$ such that $u c \bar{u}=i$ and let the automorphism $\Phi:=T_{u, \bar{u}}$ of $\mathbb{A}=\{\mathbb{H}, \mathbb{O}\}$, with $\Phi^{-1}=T_{\bar{u}, u}$. We have

$$
\begin{aligned}
\Phi \circ T_{c, \bar{c}} \circ \Phi^{-1} & =T_{u, \bar{u}} \circ T_{c, \bar{c}} \circ T_{\bar{u}, u} \\
& =T_{u c \bar{u} u \overline{c u}} \\
& =T_{i, \bar{i}}
\end{aligned}
$$

Then $\mathbb{A}_{T_{c, \bar{c}}}$ and $\mathbb{A}_{T_{i \bar{i}}}$ are isomorphic (Theorem 2.1) and (Diouf, 2017), we have $\mathbb{H}_{T_{i \bar{i}}}$ is isomorphic to $\mathbb{H}(i, 1)$.

I's clear that if $\operatorname{dim}(A) \geq 2$, theirs algebras can be obtained by using the duplication process.

Corollary 1 Let A be an absolute valued algebra containing two elements e and c. The following assertions are equivalent:

1. e is left unit and c central orthogonal to $e$,

2. A is isomorphic to $\mathbb{C}, \mathbb{H}_{i}$ or $\mathbb{O}_{i}$.

Definition 2 An element e is called generalyzed left unit if it satisfies to $\left[L_{e}, L_{x}\right]=0$, for all $x$ in $A$ (Chandid \& Rochdi, 2008).

We give a generalisation of the papier (Diankha, et al., 2013).

Theorem 5 Let $A$ be an finite dimensional absolute valued algebra contains generalized left unit e and central element $c$. Then $A$ is precisely $\mathbb{R}, \mathbb{C}, \mathbb{H}(a, b)$ or $\mathbb{O}_{c}$.

Proof. If $\operatorname{dim}(A) \in\{1,2,8\}$, then $A$ is isomorphic to $\mathbb{R}, \mathbb{C}, \mathbb{O}_{c}$ ((Diankha \& all, 2013), (Chandid \& Rochdi, 2008)). The algebras $\mathbb{H}(a, b)$ and ${ }^{\star} \mathbb{H}(a, b)$ are the unique four-dimensional absolute valued algebras containing a generalized left unit (Diouf, 2014). Without loss of generality, assume that $\|c\|=1$.

For the algebra $\mathbb{H}(a, b)$,

$$
\begin{aligned}
\text { c is central } & \Leftrightarrow x \star_{1} c=c \star_{1} x, \text { for all } x \text { in } \mathbb{H} \\
& \Leftrightarrow \text { axcb }=\text { acxb, for all } x \text { in } \mathbb{H} \\
& \Leftrightarrow x c=c x, \text { for all } x \text { in } \mathbb{H} \\
& \Leftrightarrow \quad c \in Z(\mathbb{H}) \cap S(\mathbb{R})=\{-1,1\} .
\end{aligned}
$$

Then the algebra $\mathbb{H}(a, b)$ contains a central element.

For the algebra ${ }^{\star} \mathbb{H}(a, b)$,

$$
\begin{aligned}
c \text { is central } & \Leftrightarrow x \star_{2} c=c \star_{2} x, \text { for all } x \text { in } \mathbb{H} \\
& \Leftrightarrow \bar{x} a c b=\bar{c} a x b, \text { for all } x \text { in } \mathbb{H} \\
& \Leftrightarrow \bar{x} a c=\bar{c} a x(*), \text { for all } x \text { in } \mathbb{H}
\end{aligned}
$$


For $x=1$, we have $a c=\bar{c} a$ and $(*)$ imply $\bar{x} a c=a c x(* *)$, for all $x$ in $\mathbb{H}$.

New put $x=a c$, we have $(a c)^{2}=\|a c\|^{2}=\|a\|^{2}\|c\|^{2}=1$. Hence $a c= \pm 1$ and $(* *)$ imply $\bar{x}=x$, for all $x$ in $\mathbb{H}$, which is absurd. Then the algebra $\star \mathbb{H}(a, b)$ does not contain a central element..

Proposition 1 Let $A$ be an absolute valued algebra containing a generalized left unit e and a central idempotent element $c$ such that $e \in c^{\perp}$. Then $A(e, c)$ is finite dimensional and isomorphic to $\mathbb{C}$.

Proof. The norm \|..\| of $A$ comes from an inner product and $x \mapsto x^{\star}:=2<c, x>c-x$ is an involution (El-Mallah, 1990). Without loss of generality, assume that $\|e\|=1$. We have $c e=e c=e c^{2}=c(e c)$. This implies $e c=c e=e$. The element $e$ is orthogonal to $c$, then $e^{2}=-\|e\|^{2} c=-c$ (El-Mallah, 1990).

Problem 1 Let $A$ be an absolute valued algebra containing a generalized left unit $e$ and a central element $c$. Is $A$ a finite dimensional? This problem is solved partialy if $e$ is idempotent (Calderon, et al., Preprint)..

\section{References}

Albert, A. A. (1947). Absolute valued real algebras. Ann. Math., 48, 495-501. https://doi.org/10.2307/1969182

Benslimane, M., \& Moutassim, A. (2011). Some new class of absolute valued algebras with left unit. Adv. appl. Clifford alg., 21, 31-40. https://doi.org/10.1007/s00006-010-0246-4

Bott, R., \& Milnor, J. (1958). On the parallelizability of the spheres. Bull. Amer. Math. Soc., 64, 87-89. https://doi.org/10.1090/S0002-9904-1958-10166-4

Calderón, A., Diouf, A., ..., \& Rochdi, A. Absolute valued algebras with slightly one sided unit. Preprint.

Calderon, A., Calderon, A., ..., \& Rochdi, A. (2011). Finite - dimensional absolute - valued algebras. israel journal of mathematics 184, 193 - 220.https://doi.org/10.1007/s11856-011-0065-x

Chandid, A., \& Rochdi, A. (2008). Mutations of absolute valued algebras. Int. J. Algebra, 2, 357-368.

Cuenca, J. A., Darpo, E., \& Dieterich, E. Classification of the finite dimentional absolute valued algebras having a non-zero central idempotent or a one-sided unity. To appear.

Diankha, O., Diouf, A., \& Rochdi, A. (2013). A brief statement on the absolute-valued algebras with one-sided Unit. Int. J. Algebra., 7(17), 833 - 838. https://doi.org/10.12988/ija.2013.31092

Diouf, A. (2014). Algbres absolument values ?unit?gnralise ?gauche. Thse Doctorale, Universit?Cheikh Anta Diop, Facult?des Sciences et Tchniques, Dakar.

Diouf, A. (2017). Absolute Valued Algebras With Strongly One Sided Unit. Journal Mathematics Research, 9(1), 32 35. https://doi.org/10.5539/jmr.v9n1p32

El-Mallah, M. L. (1990). Absolute valued algebras containing a central idempotent. J. Algebra128180-187.

Kervaire, M. (1958). Non-parallelizability of the n-sphere for $n>7$. Proc. Nat. Acad. Sci. USA, 44, 280-283. https://doi.org/10.5539/jmr.v9n1p32

Postnikov, M. (1985). Lecons de gomtrie. Groupes et algbres de Lie. Editions Mir, Moscow.

Ramirez, M. I. (1999). On four absolute valued algebras. Proceedings of the International Conference on Jordan Structures (Malaga, 1997), 169-173.

Rochdi, A. (2003). Eight-dimensional real absolute valued algebras with left unit whose automorphism group is trivial. IJMMS, 70, 4447-4454. https://doi.org/10.1155/s016117120320538x

Rodriguez, A. (1992). One-sided division absolute valued algebras. Publ. Math., 36, 925-954.

Rodriguez, A. (2004). Absolute valued algebras, and absolute valuable Banach spaces. Advanced courses of mathematical analysis I, 99-155, World Sci. Publ., Hackensack, NJ.

Rodriguez, A. (1994). Absolute valued algebras of degree two. In Nonassociative Algebra and its applications (Ed. S. González), 350-356, Kluwer Academic Publishers, Dordrecht-Boston-London.

Schafer, R. (1996). An Introduction to Nonassociative Algebras. Academic Press.

Urbanik, K., \& Wright, F. B. (1960). Absolute valued algebras. Proc. Amer. Math. Soc., 11, 861-866. https://doi.org/10.1090/S0002-9939-1960-0120264-6 


\section{Copyrights}

Copyright for this article is retained by the author(s), with first publication rights granted to the journal.

This is an open-access article distributed under the terms and conditions of the Creative Commons Attribution license (http://creativecommons.org/licenses/by/4.0/). 